\title{
Le calcanéus « Regourdou 2 ": étude morphométrique comparative et discussion autour de sa place dans la variabilité des Néandertaliens
}

Dany Coutinho Nogueira, Frédéric Santos, Patrice Courtaud et Christine Couture-Veschambre

\section{CpenEdition}

\section{Journals}

Édition électronique

URL : http://journals.openedition.org/paleo/3090

DOI : $10.4000 /$ paleo.3090

ISSN : 2101-0420

Éditeur

SAMRA

\section{Édition imprimée}

Date de publication : 30 décembre 2017

Pagination : 71-89

ISSN : $1145-3370$

Référence électronique

Dany Coutinho Nogueira, Frédéric Santos, Patrice Courtaud et Christine Couture-Veschambre, «Le calcanéus «Regourdou 2 » : étude morphométrique comparative et discussion autour de sa place dans la variabilité des Néandertaliens », PALEO [En ligne], 28 | 2017, mis en ligne le 01 juin 2018, consulté le 07 juillet 2020. URL : http://journals.openedition.org/paleo/3090 ; DOI : https://doi.org/ 10.4000/paleo.3090

\section{(c) (i) $\Theta($}

PALEO est mis à disposition selon les termes de la licence Creative Commons Attribution - Pas d'Utilisation Commerciale - Pas de Modification 4.0 International. 


\title{
Le calcanéus « Regourdou 2 »: étude morphométrique comparative et discussion autour de sa place dans la variabilité des Néandertaliens
}

\author{
Dany COUTINHO NOGUEIRA(a,b), Frédéric SANTOS(a), \\ Patrice COURTAUD ${ }^{(a)}$, Christine COUTURE-VESCHAMBRE ${ }^{(a)}$
}

\begin{abstract}
Résumé : Parmi les ossements découverts à Regourdou figurait un calcanéus droit, Regourdou 2, trouvé hors contexte sépulcral. Cet ossement exhumé en 1963 est régulièrement cité dans la littérature concernant les os du pied des Néandertaliens, sans avoir fait l'objet d'une étude approfondie. Dans cette contribution, nous proposons une analyse complète de cette pièce incluant une étude taphonomique, morphologique et une étude métrique et multivariée. L'ensemble de ces données permettra de discuter de son insertion dans la variabilité néandertalienne.

Si les résultats des analyses morphométriques confirment le caractère néandertalien de ce vestige, ils mettent aussi en évidence des particularités anatomiques inédites pour ce groupe (dimensions générales, proportions, morphologie des surfaces articulaires et robustesse). Cette étude apporte donc de nouvelles données sur la variabilité morphologique connue jusqu'alors pour cet ossement et ce groupe fossile. De plus, la description taphonomique apporte des informations inédites quant à son histoire et sa découverte. Enfin, du fait de son appartenance au côté droit, comme le calcanéus Regourdou 1, cet os démontre la présence d'un deuxième individu adulte dans ce site.
\end{abstract}

Mots-clés : Néandertal, Moustérien, Regourdou, calcanéus, variabilité morphométrique.

Abstract: The calcaneus "Regourdou 2": comparative morphometric study and discussion about its position in the neanderthalian variability. Among the numerous bones uncovered in the site of Regourdou, lied a right calcaneus, Regourdou 2, found out any funeral context. This remain discovered in 1963 is regularly cited in the literature regarding the Neanderthals foot bones, but it never has been precisely studied. In this paper, we propose a taphonomic, morphologic as well as a metric and multivariate analysis of this bone remain to discuss its inclusion in the Neanderthal variability.

If the morphometric results confirmed this piece belongs to a Neanderthal individual, they highlight new characters for this group (general dimensions, proportions, morphology of the articular surfaces and robusticity). This study contributes to extend the morphological variability actually known for this fossil bones and this group. The taphonomic description also helps to bring unknown data and assumptions about its history and its discovery.

Moreover, according to its affiliation to the right side, as the calcaneus Regourdou 1, this foot bone demonstrates the presence of a second adult individual in this site.

Key-words: Neanderthal, Mousterian, Regourdou, calcaneus, morphometric variability.

(a) UMR 5199 PACEA, Université de Bordeaux, CNRS, MCC ; LabEx Sciences archéologiques de Bordeaux, programme financé par I'ANR - $n^{\circ}$ ANR-10-LABX-52.

(b) EPHE - PSL Research University, Paris - Chaire d'Anthropologie biologique Paul Broca - dany.coutinho-nogueira@etu.ephe.fr 


\section{1 - Introduction}

En 2008, lors du récolement des collections au Musée National de Préhistoire aux Eyzies-de-Tayac, des restes humains sont retrouvés dans les caisses de faune du site de Regourdou parmi lesquels des fragments de la ceinture pelvienne, un important fragment de fémur, une patella, des fragments du tibia et de la fibula gauche. Ces restes appartiennent au squelette Regourdou 1 (Madelaine et al. 2008) et ils complètent parfaitement l'inventaire des pièces découvertes en septembre 1957. Dès lors, le squelette de Regourdou 1 fait l'objet d'une révision globale. À ce jour, les travaux ont porté sur plusieurs régions anatomiques : la mandibule (Piveteau 1963-1964), le sternum (Vallois 1965 ; Gómez-Olivencia et al. 2012), les membres supérieurs et les clavicules (Vandermeersch et Trinkaus 1995 ; Volpato et al. 2005, 2006, 2011), le bassin (Meyer et al. 2011) et les vertèbres (Piveteau 1966) reprises dans une étude globale avec les côtes (Gómez-Olivencia et al. 2013).

Parmi les ossements du pied découverts en 1957, et auxquels s'ajoute un naviculaire droit retrouvé en 2008 (Madelaine et al. 2008), seuls les talus ont fait l'objet d'une étude approfondie (Gambier 1981, 1982). Les autres os ont été ponctuellement utilisés dans le cadre de travaux divers (Trinkaus 1975, 1996 ; Rhoads et Trinkaus 1977 ; Schmitt 1998 ; Raichlen, Armstrong et Lieberman 2011 ; Pablos et al. 2014, 2015) et le naviculaire droit est totalement inédit.

L'inventaire (tabl. 1) met en évidence une représentation différente entre les deux côtés, le pied droit étant plus complet dans son ensemble que le pied gauche, surtout au niveau du métatarse. Le tarse est assez mal conservé avec seulement 3 pièces à droite (talus, calcanéus et naviculaire) et 2 pièces à gauche (talus et calcanéus). Les deux talus, droit et gauche, et le calcanéus droit proviennent des découvertes de 1957 et ils font partie de l'inventaire des restes du squelette Regourdou 1 conservés au Musée d'Art et d'Archéologie du Périgord. Le calcanéus gauche a été trouvé récemment dans la collection de la famille Constant (Maureille et al. 2015a). Cette pièce ne présente pas ou plus de marquage. Les caractéristiques morphométriques de ce calcanéus le rapprochent de la pièce droite ; il peut en être le symétrique (Maureille et al. 2015a).

Du point de vue de la répartition des restes dans le site, d'après le marquage préservé sur ces différentes pièces du tarse, il semblerait que la majorité des os des deux pieds proviennent du même carré G3, à proximité immédiate du carré G2 dans lequel l'essentiel des restes humains ont été retrouvés (Maureille et al. 2015b). Quelques pièces sont trouvées dans le carré F3 adjacent; une seule pièce est issue du carré E1 (une phalange proximale) et du carré F2 (le naviculaire droit, fig. 1).

Plusieurs études font état de la présence d'un deuxième individu "Regourdou 2 ». Cette dénomination apparaît pour la première fois dans le travail d'E. Trinkaus (1975) et concerne un calcanéus droit. Les données métriques correspondent cependant au calcanéus Regourdou 1. En 1995, B. Vandermeersch et E. Trinkaus mentionnent de nouveau la présence d'un second individu qu'ils identifient sur la base d'une mauvaise congruence articulaire des ossements du tarse postérieur de Regourdou 1. En 1998, A. Schmitt, dans son étude sur les calcanéus néandertaliens, mentionne la présence d'un deuxième calcanéus droit sur le site et le nomme également "Regourdou 2 ». Depuis, cette pièce est régulièrement citée dans la littérature (Madelaine et al. 2008 ; Raichlen, Armstrong et Lieberman 2011 ; Pablos et al. 2014 ; Maureille et al. 2015a) sans avoir fait l'objet d'une étude précise. L'objectif de notre travail est donc de faire une présentation complète de cette pièce inédite et de ses caractéristiques morphométriques afin de discuter de son insertion dans la variabilité néandertalienne.

\section{2 - Matériel et méthodes}

\section{Calcanéus Regourdou 2}

II s'agit d'un calcanéus droit d'aspect particulièrement massif, nettement plus volumineux que le calcanéus droit

\begin{tabular}{|l|l|l|}
\hline Inventaire des os du pied & \multicolumn{1}{|c|}{ Pied droit (localisation) } & \multicolumn{1}{|c|}{ Pied gauche (localisation) } \\
\hline Tarse & $\begin{array}{l}\text { Talus (G3) } \\
\text { Calcanéus (G1?) } \\
\text { Naviculaire (F2) }\end{array}$ & $\begin{array}{l}\text { Talus (G3) } \\
\text { Calcanéus }\end{array}$ \\
\hline Métatarse & MTT III (G3) - MTT IV (G3) - MTT V (F3) & -- \\
\hline Phalanges & $\begin{array}{l}\text { 1 phalange proximale (E1) } \\
\text { 1 phalange moyenne (G3) } \\
\text { 2 phalanges distales (dont celle de } \\
\text { I'hallux) (G3 et F3 pour l'hallux) }\end{array}$ & $\begin{array}{l}\text { 3 phalanges proximales (dont celle } \\
\text { de l'hallux) (G3) } \\
\text { 4 phalange moyenne ?? } \\
\text { l'hallux) (F3 et G3) }\end{array}$ \\
\hline
\end{tabular}

Tableau 1 - Inventaire des os du pied découverts à Regourdou (Regourdou 1). Entre parenthèses figure la référence de l'aire de fouille d'où sont issus les vestiges.

Table 1 - Inventory of the foot bones discovered at Regourdou (Regourdou 1). Between parentheses the square area reference from where the remains come. 

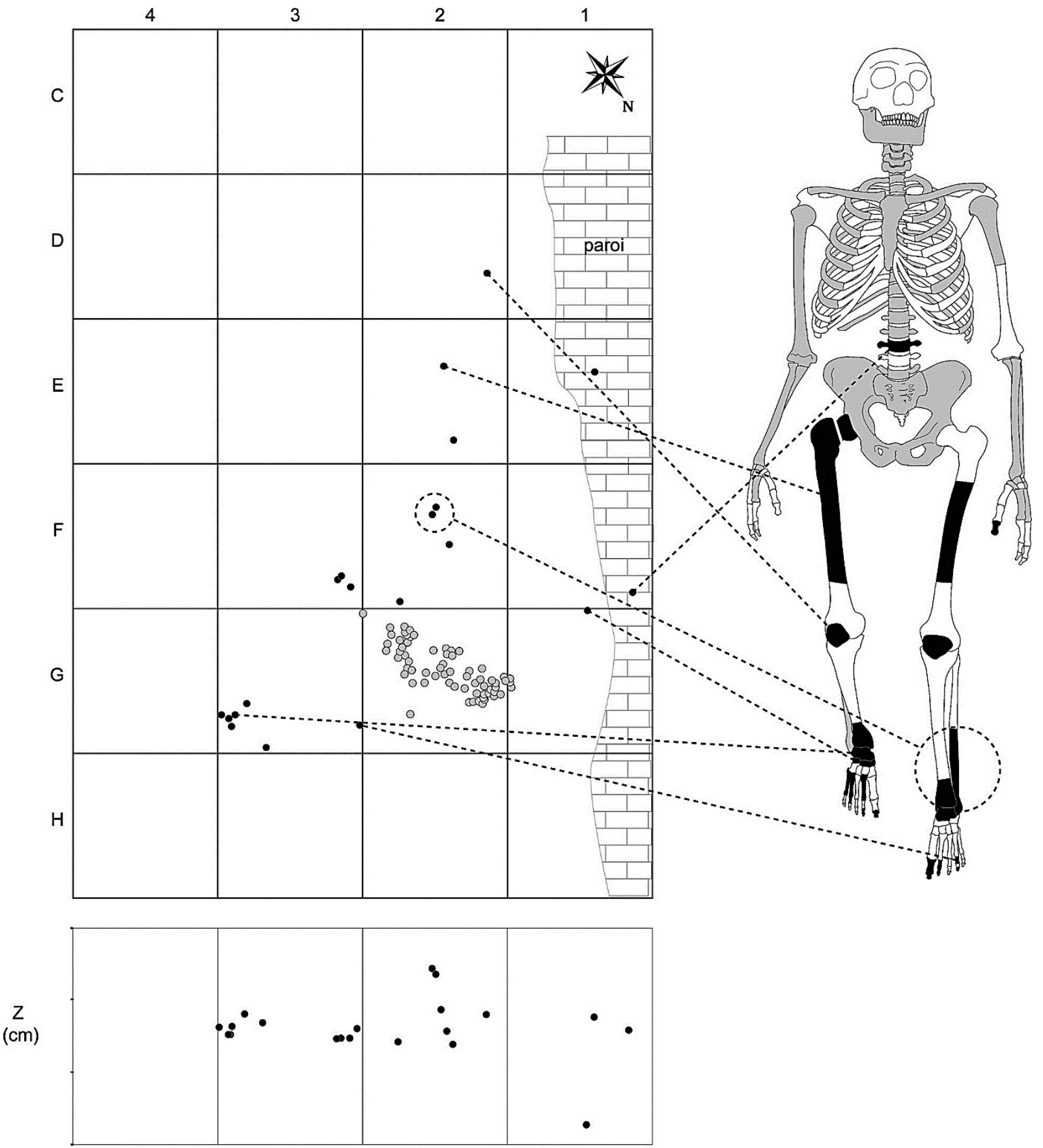

Figure 1 - Localisation des restes osseux, découverts en 2007, du site de Regourdou (Maureille et al. 2015b).

Figure 1 - Location of the bone remains, discovered in 2007, in the site of Regourdou (Maureille et al. 2015b).

de Regourdou 1. Cette pièce osseuse, du fait de son appartenance au côté droit, atteste donc de la présence d'un deuxième individu dans le site. Le calcanéus Regourdou 2 présente un bon état de conservation général malgré des pertes de substance osseuse, en particulier sur la face latérale.

II n'est malheureusement pas marqué et sa localisation précise dans le site n'est pas connue. II a été trouvé à proximité de la paroi du gisement opposée à celle de la sépulture. II appartiendrait à la couche 4 et proviendrait d'une zone de $4 \mathrm{~m}^{2}$ particulièrement bréchifiée (carrés K8, K9, L8 et L9), ultérieurement fouillée en 1963 (Maureille, comm. pers).

\section{Matériel de comparaison}

L'échantillon de comparaison utilisé dans cette étude est composé de Néandertaliens d'Europe et du Proche-Orient 
ainsi que des individus de La Sima de los Huesos, d'Hommes anatomiquement modernes du Paléolithique moyen et supérieur et d'un échantillon d'individus provenant de séries archéologiques d'époques historiques. Afin de permettre les comparaisons avec le calcanéus Regourdou 2, ces spécimens ont été sélectionnés en fonction de leur état de conservation général et de leur âge, tous les individus étant adultes. Lorsque les deux ossements étaient disponibles, le côté droit a été choisi de préférence.

L'échantillon néandertalien est composé de 10 individus d'Europe et du Proche-Orient. Notre étude inclut également 10 individus du site de La Sima de los Huesos en Espagne. Ces derniers sont généralement considérés comme appartenant à la lignée néandertalienne (Arsuaga et al. 1991 ; Martínez et Arsuaga 1997). De plus, les travaux de
Pablos et al. (2014) rapprochent morphologiquement et métriquement les calcanéus de La Sima de los Huesos de ceux des néandertaliens.

Les données métriques ont pu être recueillies sur les spécimens originaux pour Regourdou 1 et 2 , sur des moulages présents au laboratoire PACEA (La Ferrassie 1 et 2, La Chapelle-aux-Saints et Spy 2), les autres données proviennent de la bibliographie (tabl. 2).

Le groupe d'Hommes modernes du Paléolithique moyen et supérieur est constitué de 24 individus provenant d'Europe, du Proche-Orient et d'Asie orientale. Les données métriques sont entièrement bibliographiques (tabl. 3).

Notre échantillon subactuel est composé de 146 individus provenant du cimetière du couvent des sœurs grises de Beauvais (Fémolant 1992 ; Chevalier 1993), des nécropoles de Jau-Dignac et Loirac (Cartron et Castex

\begin{tabular}{|c|c|c|c|c|c|}
\hline & Site & Origine & Latéralisation & Stade isotopique & Auteurs \\
\hline \multirow{10}{*}{ Néandertaliens } & Regourdou 1 & France & Droit & OIS 5 & Lemée (2008 inédit) \\
\hline & La Chapelle-aux-Saints & France & Gauche & OIS 3 & Boule (1911-1913) \\
\hline & La Ferrassie 1 & France & Droit & \multirow{2}{*}{ Fin OIS 3} & \multirow{2}{*}{ Heim (1982) } \\
\hline & La Ferrassie 2 & France & Droit & & \\
\hline & Spy 2 & Belgique & Droit & OIS 3 & Fraipont et Lohest (1887) \\
\hline & Kiik-Koba 1 & Ukraine & Droit & Fin OIS 5/ début OIS 4 & Endo (1970) et Heim (1978) \\
\hline & Tabun C1 & Israël & Droit & Fin OIS 6 ou début OIS 5 & McCown et Keith (1939) \\
\hline & Amud 1 & Israël & Gauche & \multirow{3}{*}{ Transition OIS 4/OIS 3} & Endo (1970) \\
\hline & Shanidar 1 & Irak & Droit & & \multirow{2}{*}{ Trinkaus (1983b) } \\
\hline & Shanidar 3 & Irak & Droit & & \\
\hline \multirow{10}{*}{ Sima de los Huesos } & AT-319 & Espagne & Droit & \multirow{10}{*}{ Fin OIS 12 / début OIS 11} & \multirow{10}{*}{ Pablos et al. (2014) } \\
\hline & AT-489 & Espagne & Droit & & \\
\hline & AT-663 & Espagne & Gauche & & \\
\hline & AT-969 & Espagne & Droit & & \\
\hline & AT-981 & Espagne & Droit & & \\
\hline & AT-1576 & Espagne & Droit & & \\
\hline & AT-1740 & Espagne & Gauche & & \\
\hline & AT-2466 & Espagne & Droit & & \\
\hline & AT-2741 & Espagne & Droit & & \\
\hline & AT-3130 & Espagne & Droit & & \\
\hline
\end{tabular}

Tableau 2 - Inventaire des calcanéus néandertaliens utilisés comme échantillon de comparaison.

Table 2 - Inventory of the neanderthalian calcaneus included as comparison sample. 


\begin{tabular}{|c|c|c|c|c|}
\hline Site & Origine & Latéralisation & Stade isotopique & Auteurs \\
\hline Abri Pataud & France & Gauche & \multirow{3}{*}{ OIS 2} & Billy (1975) \\
\hline Cap-Blanc & France & Droit & & Bonin (1935) \\
\hline Chancelade & France & Gauche & & Billy (1969) \\
\hline Cro Magnon 4336 & France & Droit & Début OIS 2 & Schmitt (1998) \\
\hline Oberkassel & Allemagne & Droit & Fin OIS 2 & Bonnet (1919) \\
\hline Paviland & Pays de Galles & Droit & Fin OIS 3-Début OIS 2 & Trinkaus (2000) \\
\hline Dolni Vestonice 3 & République Tchèque & Gauche & \multirow{8}{*}{ OIS 2} & \multirow{4}{*}{ Sladek et al. (2000) } \\
\hline Dolni Vestonice 13 & République Tchèque & Droit & & \\
\hline Dolni Vestonice 15 & République Tchèque & Droit & & \\
\hline Dolni Vestonice 16 & République Tchèque & Droit & & \\
\hline Predmosti III & République Tchèque & Droit & & \multirow{4}{*}{ Matiegka (1938) } \\
\hline Predmosti IX & République Tchèque & Droit & & \\
\hline Predmosti X & République Tchèque & Droit & & \\
\hline Predmosti XIV & République Tchèque & Droit & & \\
\hline Sungir 1 & Russie & Droit & Début OIS 2 & Trinkaus et al. 2014 \\
\hline Ohalo II & Israël & Droit & OIS 2 & $\begin{array}{l}\text { Trinkaus (données non } \\
\text { publiées) }\end{array}$ \\
\hline Qafzeh 3 & Israël & Gauche & \multirow{4}{*}{ OIS 5} & \multirow{3}{*}{ Vandermeerch (1981) } \\
\hline Qafzeh 8 & Israël & Gauche & & \\
\hline Qafzeh 9 & Israël & Droit & & \\
\hline Skhul IV & Israël & Droit & & McCown et Keith (1939) \\
\hline Tianyuan & Chine & Droit & OIS 3 & Shang et Trinkaus (2010) \\
\hline Minatogawa 3 & Japon & Droit & \multirow{3}{*}{ Fin OIS 2} & \multirow{3}{*}{ Baba et Endo (1982) } \\
\hline Minatogawa 4 & Japon & Droit & & \\
\hline Minatogawa 1 & Japon & Gauche & & \\
\hline
\end{tabular}

Tableau 3 - Inventaire des calcanéus des Hommes anatomiquement modernes du Paléolithique Moyen et Supérieur utilisés comme échantillon de comparaison.

Table 3 - Inventory of the Middle and Upper Palaeolithic Homo sapiens calcaneus included as comparison sample. 
2006) et de Seyssel-Albigny (Bizot et Serralongue 1988) et de la série archéologique mamelouke de Tel Dan en Israël (Courtaud, données non publiées) ainsi que de populations d'Amérindiens Libben et d'Egyptiens Keneh (Trinkaus 1975 ; tabl. 4).

Ces deux derniers groupes constituent ensemble notre échantillon d'Hommes anatomiquement modernes.

\section{Méthodes}

Peu d'informations sur l'histoire de la découverte de ce vestige nous sont parvenues. Dans ce travail, une étude taphonomique a été effectuée afin d'extraire de nouvelles données et indices permettant de retracer l'histoire de cette pièce (aspects de surface, état de conservation, position au moment de la découverte, atteintes péri et post-mortem, etc.). Une étude descriptive morphologique et comparative a également été réalisée pour mettre en évidence les principaux caractères de cette pièce inédite. Enfin, afin de comparer ce fossile avec nos échantillons de référence et de le replacer dans la variabilité néandertalienne, une analyse métrique et multivariée a été effectuée.

\section{Étude métrique et multivariée}

Afin de pouvoir comparer nos données avec d'autres études, nous avons utilisé les mesures les plus couramment reprises dans la littérature définies par Martin et Saller (1956) et compilées par Bräuer (1988), ainsi que la mesure T1 (longueur du processus médial) définie par Trinkaus (1975) (Annexe 1).

Les données sont synthétisées sous la forme de boxplots ou " boites à moustaches ». Pour chaque variable, un test de Kruskal-Wallis est mené afin de comparer les médianes des quatre populations étudiées. Si ce test révèle des différences significatives, des comparaisons post-hoc sont effectuées en utilisant la correction de Hochberg pour les $p$ valeurs. Nous utilisons pour cela le package $R$ agricolae (De Mendiburu 2017).

Nous avons effectué des analyses en composantes principales afin de mettre en évidence d'éventuels rapprochements entre individus et/ou groupes.

Dans le cas des études biologiques, les variables peuvent être très fortement corrélées entre elles, l'ACP a alors tendance à répartir la majorité des données sur un seul axe sous l'effet d'un facteur, la taille. Afin de s'affranchir de cet effet, un double centrage des données peut être effectué, permettant alors de ne conserver que les informations relatives à la conformation géométrique (Cibert et al. 1999). En premier lieu, une fonction représentant la taille des individus doit être définie. Le choix classique consiste à calculer, pour chaque individu, la moyenne arithmétique de toutes les variables. Afin de lisser les différences d'ordre de grandeur entre ces variables, le tableau est préalablement log-transformé. Enfin, on effectue un centrage en lignes en retranchant à chaque valeur du tableau la taille de l'individu correspondant : on ramène ainsi la moyenne de chaque ligne (i.e. la taille de chaque individu) à une même valeur, qui est égale à 0 .

Dans le cas des calcanéus, la taille des spécimens est fortement corrélée au sexe (Steele 1976 ; Introna et al. 1997 ; Bidmos et Asala 2003, 2004) ; le double centrage permet donc de s'affranchir également d'un effet lié au sexe des individus et paraît donc adapté à ce type de matériel. Seules les variables les plus représentées dans nos échantillons sont retenues afin de réaliser ces ACP. Les principaux paramètres du calcanéus : la longueur, la largeur, la hauteur et les dimensions des surfaces articulaires sont représentés par ces variables.

Les boxplots sont une forme de représentation graphique permettant de mettre en évidence la distribution d'une série statistique quantitative grâce à la médiane et aux quartiles (25\% et $75 \%$ de l'échantillon). Cet outil est particulièrement utile pour comparer, pour une même variable, plusieurs populations de tailles différentes.

\section{3 - Résultats}

\section{Étude taphonomique}

Le calcanéus Regourdou 2 présente un bon état de conservation. L'os est presque complet mais des altérations plus ou moins étendues sont visibles sur toutes les faces. II est particulièrement concrétionné (sur sa face médiale), minéralisé et lourd. Sa couleur est beige avec des nuances plus foncées. Son poids est de $82,7 \mathrm{~g}$.

La face dorsale (fig. 2a) présente des lacunes osseuses laissant apparaître l'os spongieux sous-jacent, principalement au niveau de la tubérosité postérieure. Ces atteintes sont globalement superficielles, à l'exception d'une entaille profonde du côté médial, qui sera plus

\begin{tabular}{|c|c|c|c|c|c|c|c|}
\hline & $\begin{array}{c}\text { Beauvais } \\
\text { (France) }\end{array}$ & $\begin{array}{c}\text { Jau-Dignac et } \\
\text { Loirac } \\
\text { (France) }\end{array}$ & $\begin{array}{c}\text { Seyssel- } \\
\text { Albigny } \\
\text { (France) }\end{array}$ & Tel Dan (Israël) & $\begin{array}{c}\text { Libben } \\
\text { (Etats-Unis) }\end{array}$ & $\begin{array}{c}\text { Keneh } \\
\text { (Egypte) }\end{array}$ & total \\
\hline Période & $\begin{array}{c}15 \mathrm{e}-18 \mathrm{e} \text { siècles } \\
\text { ap. J.-C. }\end{array}$ & $\begin{array}{c}7 \mathrm{e}-11 \mathrm{e} \text { et 12e- } \\
18 \mathrm{e} \text { siècles ap. } \\
\text { J.-C. }\end{array}$ & $\begin{array}{c}5 \mathrm{e}-7 \mathrm{e} \text { siècles } \\
\text { ap. J.-C. }\end{array}$ & $\begin{array}{c}15 \mathrm{e}-16 \mathrm{e} \text { siècles } \\
\text { ap. J.-C. }\end{array}$ & $\begin{array}{c}7 \mathrm{e} 11 \mathrm{e} \text { siècles } \\
\text { ap. J.-C. }\end{array}$ & $\begin{array}{c}3150-3050 \text { av. } \\
\text { J.-C. }\end{array}$ & \\
\hline $\begin{array}{c}\text { Nombre } \\
\text { d'individus }\end{array}$ & 14 & 14 & 8 & 20 & 40 & 50 & 146 \\
\hline
\end{tabular}

Tableau 4 - Inventaire des séries subactuelles utilisées comme échantillon de comparaison.

Table 4 - Inventory of the modern archaeological series included as comparison sample. 


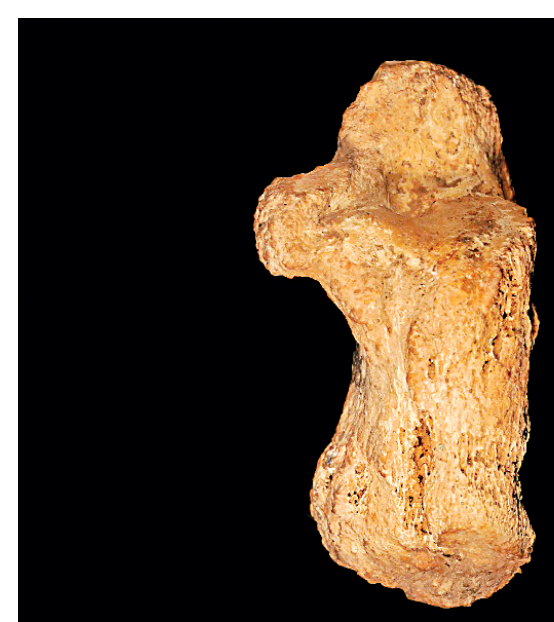

a

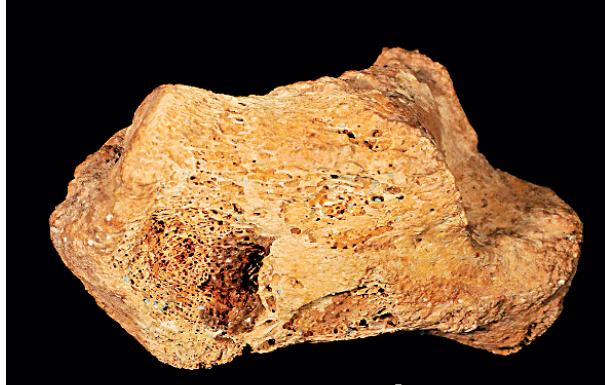

C

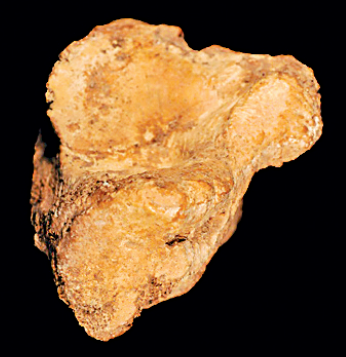

$\mathbf{e}$

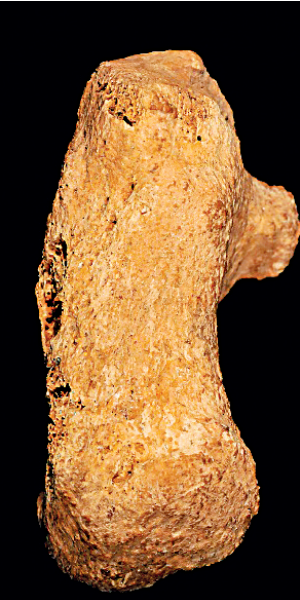

b

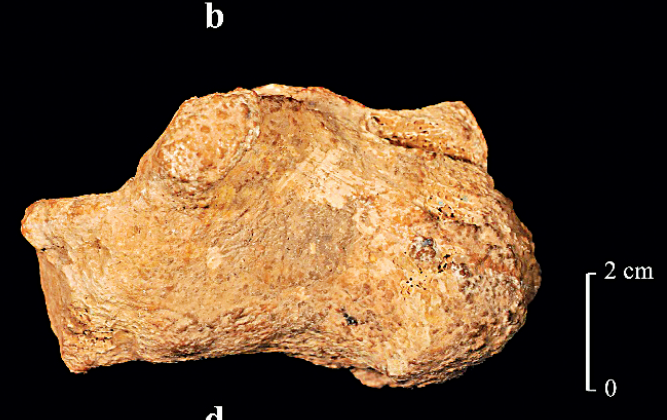

d

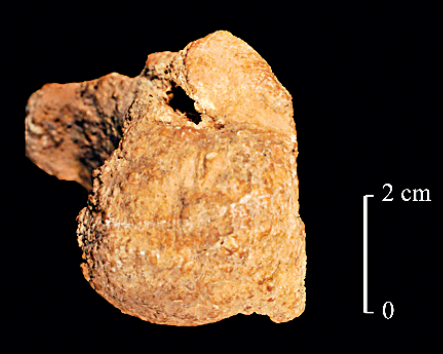

f
Figure 2 - Le calcanéus Regourdou 2 (a : face dorsale, $b$ : face plantaire, $c:$ face latérale, $d:$ face médiale, e : face antérieure, $f:$ face postérieure) OPatrice Courtaud et Gauthier Devilder.

Figure 2 - The Regourdou 2 calcaneus (a: dorsal face, b: plantar face, c: lateral face, $d$ : medial face, e: anterior face, $f$ : posterior face) CPatrice Courtaud and Gauthier Devilder. précisément décrite ci-après. La partie antérieure, qui porte les surfaces articulaires, est en revanche intacte.

La face plantaire (fig. 2b) est particulièrement bien conservée, des pertes mineures et superficielles de substance osseuse sont toutefois à noter dans la partie antérieure à proximité immédiate de la surface articulaire cuboïdienne.

La face latérale (fig. 2c) est plus claire car elle ne montre pas de concrétion. Elle est particulièrement dégradée dans sa partie postérieure avec une disparition importante de l'os cortical laissant l'os spongieux totalement découvert (hauteur : $36,2 \mathrm{~mm}$, longueur : $24,9 \mathrm{~mm}$ ). Cet état de surface est particulièrement fréquent dans le registre fossile (Amud, Endo et Kimura 1970 ; La Ferrassie 1 et 2, Heim 1982 ; Kiik-Koba, Trinkaus, Maley et Buzhilova 2008 ; La
Chapelle-aux-Saints, Boule 1911-1913 ou encore Regourdou 1, Lemée 2008), cette face étant la plus fragile.

$\mathrm{Au}$ centre de cette face, les trabécules présentent une coloration plus sombre. II ne s'agit néanmoins pas de traces de combustion. Deux autres lacunes, l'une supérieure et l'autre inférieure, moins importantes sont présentes sur la partie centrale de l'os. Elles mesurent respectivement $20,4 \mathrm{~mm}$ sur $15 \mathrm{~mm}$ et $21 \mathrm{~mm}$ sur $14 \mathrm{~mm}$. Des résidus de moulages (plastiline) correspondant à de petites inclusions de matière verte au sein des trabécules osseuses sont visibles.

Sur sa face médiale (fig. 2d), plus particulièrement dans sa partie postérieure et au niveau du sustentaculum tali, nous pouvons noter la présence de concrétion en « chou-fleur ». Ces concrétions se forment à l'air libre dans des cavités 
naturelles actives. En effet, la production ce type de concrétion est favorisée par trois facteurs : la présence de gouttelettes d'eau issue d'éclaboussures ; d'une importante évaporation et d'un dégazage du CO2 présent dans l'eau (Vanghi, Frisia et Borsato 2017). La face médiale du calcanéus a donc été en surface pendant une période prolongée, la pièce reposant probablement sur sa face latérale. La présence d'eau et le contact avec le sédiment pourraient expliquer les lacunes osseuses de la face latérale, l'eau pouvant causer une fragilisation de l'os (Hare 1980).

La face médiale du calcanéus est généralement mal conservée dans le registre fossile (Amud, Endo et Kimura 1970 ; La Ferrassie 1 et 2, Heim 1982). Or, dans le cas de Regourdou 2, l'os cortical est peu affecté par l'érosion, les concrétions ayant certainement contribué à cette bonne conservation. Néanmoins, d'autres atteintes taphonomiques sont notables sur cette face.

Elle présente une importante entaille de direction antéropostérieure dans sa partie supérieure. Ses bords ne sont pas émoussés, il s'agirait donc d'une entaille post-mortem. Cette atteinte s'étend sur $43 \mathrm{~mm}$ avec une profondeur allant croissant de l'extrémité antérieure $(2 \mathrm{~mm})$ vers l'extrémité postérieure $(8 \mathrm{~mm})$. Sa largeur évolue respectivement de $5 \mathrm{~mm}$ à $6,7 \mathrm{~mm}$. Son aspect évoque l'utilisation d'avant en arrière d'un outil tranchant de type piochon ou bien truelle (Marin-Monfort, Suñer, FernandezJalvo 2017).

Sous cette lacune, deux perforations, globalement circulaires sont espacées de $5,3 \mathrm{~mm}$. Le diamètre moyen de la lacune supérieure est de $8 \mathrm{~mm}$, alors que celui de la lacune inférieure est de $4 \mathrm{~mm}$. Cette dernière est toutefois plus profonde, $3,5 \mathrm{~mm}$, contre $2,7 \mathrm{~mm}$. Leur périphérie montre de fines ébréchures. Leur aspect est caractéristique d'atteintes post-mortem qui auraient pu être causées lors de la fouille.

Trois fines stries parallèles, sub-verticales, de $10 \mathrm{~mm}$ de long sont visibles au centre de cette face à proximité du sustentaculum tali, en arrière de la zone de passage du tendon du muscle fléchisseur de l'hallux. Elles sont manifestement accompagnées d'écaillures superficielles de la fine couche de concrétion. A cet endroit, l'os apparaît plus clair. Dans ce cas également, elles se sont produites alors que l'os était à l'état sec.

La face antérieure (fig. 2e) est quasiment intacte. Des érosions mineures et superficielles sont toutefois visibles dans la partie inférieure du pourtour de la surface articulaire distale. La face postérieure (fig. 2f) est concrétionnée. Apparaît dans sa partie supéro-médiale, la lacune décrite sur la face médiale.

\section{Description morphologique}

\section{Face dorsale (fig. 2a)}

La moitié antérieure de la face dorsale porte les surfaces articulaires talaires. Ces deux surfaces sont bien délimitées et plutôt planes. Les surfaces antérieure et moyenne sont nettement séparées l'une de l'autre, contrairement à ce qui est observé sur la plupart des calcanéus néandertaliens (Schmitt 1998). En effet, seuls les calcanéus droits de Shanidar 3 et de Spy 2 pourraient posséder également cette morphologie (la facette antérieure est bien délimitée mais la région de la facette médiale n'est pas conservée dans les deux cas). Pour le calcanéus gauche de Shanidar 3 les facettes sont fusionnées. En revanche sur Kiik-Koba, la surface antérieure est absente (Trinkaus 1975).

Entre la surface talaire moyenne et la surface talaire postérieure se trouve le sillon calcanéen qui s'élargit du bord médial vers le bord latéral. A l'intérieur de ce sillon passe le ligament interosseux talo-calcanéen. Ce sillon est profond mais étroit sur Regourdou 2 alors qu'il est large à La Chapelle-aux-Saints, La Ferrassie 1, La Ferrassie 2, Spy 2 et Kiik-Koba 1.

La surface articulaire talaire postérieure est très nette, ovalaire et convexe, à grand axe oblique en avant et latéralement. Cette surface est bien délimitée vers l'avant et latéralement, mais elle est incomplète vers l'arrière.

La moitié postérieure de la face dorsale est rugueuse, concave dans le sens antéro-postérieur et convexe dans le sens médio-latéral.

\section{Face plantaire (fig. 2b)}

Elle est très étroite et marquée par la présence, en arrière, d'une volumineuse tubérosité calcanéenne avec un processus médial (insertion des $\mathrm{m}$. abducteur de l'hallux et court fléchisseur des orteils) massif et un processus latéral (insertion du $\mathrm{m}$. abducteur du petit orteil) plus étroit mais en relief saillant. En avant, le tubercule calcanéen forme un relief saillant bien net. Entre le tubercule antérieur et la tubérosité postérieure, la face plantaire est concave dans le sens transversal et légèrement convexe dans le sens médio-latéral.

\section{Face latérale (fig. 2c)}

La face latérale présente une abrasion très importante dans le tiers postérieur, laissant apparaître l'os spongieux. La trochlée fibulaire est cassée mais son volume ne semble pas avoir été important. Elle ne semble pas avoir été très saillante contrairement à d'autres caractères observés sur cet os. Au-dessus de la trochlée fibulaire, le sillon supérieur dans lequel glisse le tendon du $\mathrm{m}$. court fibulaire est vaste et profond. En revanche, en-dessous de la trochlée, le sillon inférieur dans lequel glisse le tendon du $\mathrm{m}$. long fibulaire est très peu marqué, mal délimité et peu profond.

\section{Face médiale (fig. 2d)}

L'os est bien conservé sur cette face. Elle est très concave dans sa partie médiane. À l'avant, le sustentaculum tali est très épais, saillant et, à l'arrière, le processus médial de la tubérosité calcanéenne est bien visible montrant une extension nette de cette partie.

\section{Face antérieure (fig. 2e)}

La face antérieure est peu étendue, elle est entièrement occupée par la surface articulaire cuboïdienne. C'est une 
articulation en selle qui, sur cet individu, ne présente pas les caractéristiques typiques de ce genre d'articulation. En effet, la concavité de haut en bas est peu marquée. Il en est de même pour la convexité médio-latérale qui est très effacée. Dans son ensemble, cette surface apparaît plutôt plate sauf dans son extrémité supérieure marquée par le surplomb du rostrum du calcanéus qui prolonge la face supérieure. Ce surplomb n'est pas très accentué.

\section{Face postérieure (fig. 2f)}

Plus large en bas qu'en haut, elle est particulièrement massive et présente, de haut en bas, trois zones distinctes : - la zone supérieure, plutôt réduite en dimension, est assez lisse et répond à la bourse du tendon calcanéen ;

- la zone moyenne est très irrégulière, d'importantes rugosités et des stries verticales sont visibles, elle forme également un relief très accentué vers l'arrière. Elle correspond à l'insertion du tendon calcanéen ;

- la zone inférieure beaucoup moins lisse que la zone supérieure et plus régulière que la zone moyenne.

Il est intéressant de noter, sur cette face, la petite étendue de la zone supérieure et, inversement, le développement très massif de la zone moyenne, caractères auxquels s'ajoute le développement très accentué de la région d'insertion du tendon calcanéen. Cette région est particulièrement massive et forme une véritable protubérance vers l'arrière, cette particularité ne semble pas décrite dans la littérature. La comparaison avec le calcanéus Regourdou 1 met en évidence de façon très nette le développement exagéré de cette partie. Contrairement à Regourdou 1 caractérisé par une surface supérieure lisse et assez étendue (plus étendue que la partie inférieure légèrement dentelée), Regourdou 2 présente des proportions inverses avec une surface supérieure très réduite et une partie inférieure très développée.

\section{Étude métrique}

\section{Dimensions générales}

Les longueurs maximale et totale (M1 et M1a) sont élevées et dépassent très largement celles de tous les individus de notre échantillon de comparaison, Néandertaliens, hommes modernes et de La Sima de los Huesos (SH). Cela peut être mis en relation avec la longueur du corps du calcanéus (M5) particulièrement importante (cf. infra). En revanche, la largeur médiane (M2) se situe légèrement en dessous de la moyenne néandertalienne et de $\mathrm{SH}$ mais elle est supérieure à la plupart des individus modernes (tabl. 5). L'indice de la largeur médiane (M2/M1) est très faible. II est le plus faible de notre échantillon néandertalien et de $\mathrm{SH}$ et parmi les valeurs les plus basses des échantillons modernes.

\section{Corps du calcanéus}

La largeur minimale (M3) est estimée à $30 \mathrm{~mm}$ pour Regourdou 2, ce qui le place, avec le calcanéus droit de Spy 2 parmi les valeurs les plus importantes de nos échantillons (tabl. 5). L'indice (M3/M1) est cependant faible en comparaison avec les autres individus néandertaliens et de SH.

La hauteur totale (M4) est la plus importante de tout notre échantillon de référence, elle est par ailleurs réduite en raison de légères pertes osseuses sur la partie postérieure de la face dorsale.

\begin{tabular}{|c|c|c|c|c|c|c|c|c|c|c|c|c|c|c|c|c|c|}
\hline & & M1 & M1a & M2 & M3 & M4 & M5 & M6 & M7 & M8 & M9 & M10 & M11 & M12 & M13 & M14 & T1 \\
\hline \multicolumn{2}{|c|}{ Regourdou 2} & 90,6 & 85,6 & 44,3 & 30 & 46,4 & 67,5 & 13 & 50,7 & 32,3 & 28 & 24,2 & & 27 & & 55 & 27,5 \\
\hline \multirow{6}{*}{ Néandertaliens } & Moyennes & 76,9 & 74,1 & 43,8 & 28,4 & 41,6 & 58,7 & 15,2 & 46,1 & 31,7 & 31,9 & 24,5 & 5,6 & 26,5 & 24,9 & 43 & 26,7 \\
\hline & \begin{tabular}{|l|} 
Médiane \\
\end{tabular} & 76 & 74 & 44,3 & 28 & 42 & 59 & 14,5 & 46,1 & 31,2 & 31,9 & 24,9 & 5,5 & 26,5 & 23 & 47 & 25 \\
\hline & $\mathrm{N}$ actif & 8 & 5 & 8 & 5 & 7 & 9 & 7 & 6 & 5 & 9 & 8 & 5 & 2 & 4 & 7 & 3 \\
\hline & Min & 70 & 70 & 39 & 25 & 37 & 51 & 12 & 40 & 28,9 & 26 & 22,3 & 4,5 & 23 & 19,5 & 17 & 18 \\
\hline & Max & 83,3 & 80 & 47 & 33 & 45 & 66,1 & 19 & 51 & 38 & 37,7 & 27,2 & 7,1 & 30 & 34 & 57 & 37 \\
\hline & écart type & 5,15 & 3,96 & 2,95 & 2,97 & 2,51 & 4,86 & 2,86 & 4,06 & 3,71 & 3,9 & 1,69 & 1 & 4,95 & 6,32 & 14,04 & 9,6 \\
\hline \multirow{6}{*}{$\begin{array}{c}\text { Sima de los } \\
\text { Huesos }\end{array}$} & Moyennes & 77,4 & 74 & 43,7 & 30,8 & 38,7 & 57,2 & 17,4 & 43,5 & & 31,5 & 22,2 & 23,2 & 27,3 & 20,7 & & \\
\hline & \begin{tabular}{|l|} 
Médiane \\
\end{tabular} & 75 & 72 & 43,7 & 31,4 & 38,7 & 56,3 & 17,7 & 43,6 & & 31,7 & 22,1 & 23,3 & 27,9 & 20,1 & & \\
\hline & \begin{tabular}{|l|}
$N$ actif \\
\end{tabular} & 9 & 9 & 10 & 6 & 8 & 9 & 10 & 8 & & 10 & 10 & 10 & 9 & 8 & & \\
\hline & \begin{tabular}{|l|} 
Min \\
\end{tabular} & 72,2 & 67,4 & 39,1 & 28,1 & 36,2 & 51,6 & 12,7 & 41,5 & & 28,8 & 19,8 & 19,9 & 23,6 & 19,4 & & \\
\hline & \begin{tabular}{|l|} 
Max \\
\end{tabular} & 84,6 & 81,4 & 48,8 & 32,8 & 42,1 & 63,2 & 21,3 & 45,6 & & 35,5 & 25,3 & 27,9 & 29,9 & 23,7 & & \\
\hline & écart type & 4,9 & 5,06 & 3,17 & 2,07 & 1,72 & 4,31 & 2,69 & 1,45 & & 2,31 & 2,08 & 2,7 & 2,21 & 1,56 & & \\
\hline \multirow{6}{*}{$\begin{array}{c}\text { Paléolithique } \\
\text { moyen et } \\
\text { supérieur }\end{array}$} & moyennes & 77,1 & 69,7 & 42,5 & 25,3 & 39,6 & 54,8 & 14,1 & 43,3 & 29,8 & 30,4 & 23,2 & 4 & 26 & 23 & 47,3 & 7 \\
\hline & Mediane & 75,4 & 68,8 & 43 & 25,5 & 39,8 & 54,4 & 14,5 & 43 & 30 & 31 & 23 & 4 & 24 & 23 & 49 & 7 \\
\hline & $\mathrm{N}$ actifs & 13 & 5 & 12 & 8 & 9 & 11 & 10 & 9 & 9 & 12 & 11 & 2 & 3 & 3 & 4 & 1 \\
\hline & $\min$ & 67 & 64 & 39 & 22 & 33,5 & 45 & 11,5 & 39 & 28 & 26 & 19 & 4 & 23 & 22 & 41 & 7 \\
\hline & \begin{tabular}{|l|}
$\max$ \\
\end{tabular} & 88 & 76 & 45,9 & 30 & 45 & 62 & 15,6 & 48 & 33 & 33,8 & 27 & 4 & 31 & 24 & 50 & 7 \\
\hline & ecart type & 5,57 & 4,52 & 2,28 & 2,76 & 3,42 & 4,8 & 1,38 & 2,96 & 1,8 & 2,58 & 2,61 & 0 & 4,36 & 1 & 4,27 & \\
\hline \multirow{6}{*}{ Subactuel } & moyenne & 76 & 70,9 & 40,8 & 25,4 & 39,7 & 54 & 12,6 & 44,3 & 29,2 & 24,5 & 26,7 & 5,1 & 23,2 & 22,9 & 57,4 & 23,6 \\
\hline & Mediane & 73,4 & 69,5 & 40,1 & 25,2 & 39,2 & 52,5 & 12 & 43,8 & 29,1 & 23,4 & 26,6 & 5 & 23,4 & 22,9 & 58 & 23,3 \\
\hline & $\mathrm{N}$ actifs & 56 & 55 & 55 & 50 & 56 & 56 & 54 & 55 & 52 & 56 & 55 & 55 & 48 & 53 & 35 & 36 \\
\hline & $\min$ & 64,2 & 59,9 & 34,9 & 21,1 & 33,4 & 44,6 & 9 & 37,8 & 24,6 & 17,9 & 19,8 & 3 & 19,3 & 19,9 & 44 & 19 \\
\hline & $\max$ & 88,9 & 82,7 & 46,2 & 31,7 & 45,8 & 65,4 & 18,1 & 52,6 & 35,6 & 36,2 & 33,8 & 7,5 & 27,2 & 28,9 & 70 & 33 \\
\hline & \begin{tabular}{|l|} 
ecart type \\
\end{tabular} & 6,18 & 5,58 & 2,85 & 2,52 & 3,32 & 4,49 & 2,23 & 4,08 & 2,78 & 4,35 & 3,47 & 0,88 & 2,17 & 2,01 & 6,88 & 3,18 \\
\hline
\end{tabular}

Tableau 5 - Données métriques du calcanéus Regourdou 2 et des différents groupes populationnels.

Table 5 - Metric data of the Regourdou 2 calcaneus and of the different groups. 
Le corps du calcanéus présente dans le groupe des Néandertaliens, des longueurs (M5) qui le sépare des autres groupes (Trinkaus 1975 ; Schmitt 1998 ; Raichlen 2011 ; Pablos et al. 2014). Cette dimension est pour Regourdou 2 la plus élevée de tout notre échantillon et se situe également hors de la variabilité des groupes nonnéandertaliens (tabl. 5). L'indice M5/M1 est proche de la moyenne des Néandertaliens et de SH mais est supérieur aux groupes modernes.

\section{Tubérosité calcanéenne}

La hauteur de la tubérosité (M7) du calcanéus se situe parmi les plus importantes de nos différents groupes. Dans le groupe des Néandertaliens, seul Shanidar 1 présente une dimension supérieure. La largeur de la tubérosité (M8) est quant à elle très proche de la moyenne néandertalienne mais supérieure aux moyennes des groupes modernes (tabl. 5).

L'indice de la tubérosité calcanéenne (M8/M7) est le plus faible des six Néandertaliens pour lesquels ce calcul est possible. En ce qui concerne la longueur du processus médial (T1), Regourdou 2 se situe légèrement en deçà de la moyenne des Néandertaliens $(27,4 \mathrm{~mm})$ mais au-dessus de la moyenne actuelle $(23,6 \mathrm{~mm})$.

\section{Sustentaculum tali et surfaces articulaires}

La largeur du sustentaculum tali (M6) se situe parmi les plus faibles des différents groupes, ce qui va avec les surfaces articulaires antérieure et moyenne de petites tailles et la faible largeur médiale (M2 ; tabl. 5).

La surface articulaire postérieure étant endommagée dans sa partie médiale, la longueur (M9) de celle-ci est sousestimée, la largeur (M10) est, quant à elle, identique à la moyenne néandertalienne $(24,2 \mathrm{~mm})$. Cette dernière est supérieure à celle des individus modernes, qu'ils soient du Paléolithique $(22,7 \mathrm{~mm})$ ou subactuels $(22,8 \mathrm{~mm})$ et de $\mathrm{SH}$ (21,9 mm ; tabl. 5).

La largeur maximale de la surface articulaire cuboïdienne (M12), rarement conservée dans l'échantillon fossile (4 Néandertaliens et 5 Hommes anatomiquement modernes), est préservée sur notre spécimen. Elle est égale à la moyenne de son groupe soit $27 \mathrm{~mm}$. Elle est légèrement supérieure à la moyenne des Hommes modernes du Paléolithique supérieur et moyen $(26 \mathrm{~mm})$, la différence est plus marquée avec notre série de comparaison subactuelle (23,2 mm ; tabl. 5).

\section{Résultats de l'étude multivariée}

L'ACP avec double centrage des données a été réalisée sur toutes les populations (fossiles et subactuelles) afin d'analyser la position de Regourdou 2 par rapport aux différentes populations.

Pour cette ACP, l'axe 1 exprime 36,35\% de l'information et est principalement influencé par les variables M2, M6, M7 et M9. Le deuxième axe porte $20,52 \%$ de l'information, M5 et M10 sont les principales variables influençant cet axe (fig. 3). L'axe 3 porte $16,27 \%$ de l'information, nous n'avons pas retenu les représentations avec le troisième axe car elles n'apportaient pas de données exploitables supplémentaires.

Regourdou 2 se situe dans la variation des trois principaux groupes de cette étude. Parmi le groupe des Néandertaliens, Regourdou 2 se rapproche plus particulièrement de Kiik-Koba et d'Amud 1. Néanmoins il semble légèrement s'individualiser du reste de cette population sous l'effet conjugué des variables M5 et M10. Les populations du Paléolithique moyen et supérieur (Hommes modernes et Néandertaliens) ne sortent pas véritablement de la variation moderne. Seuls les individus de La Sima de los Huesos semblent s'individualiser principalement sous l'influence de la variable M6. L'analyse en boxplot confirme ces résultats (fig. 4), le groupe de La Sima de los Huesos présentant des données particulièrement élevées pour cette variable.

Par ailleurs, la représentation en boxplot de la variable M9 (longueur de la surface articulaire postérieure) permet de mettre en évidence une différence statistiquement significative entre le groupe des individus subactuels et chacun des trois groupes fossiles (tabl. 6). Les populations récentes présentent en effet des valeurs nettement plus faibles pour cette variable (fig. 5).

\section{7 - Discussion}

Regourdou 2 se situe pour la majorité des dimensions dans la variabilité moderne actuelle et ancienne (Paléo supérieur et moyen) comme l'ensemble des calcanéus néandertaliens et ceux de la Sima de los Huesos. II se caractérise néanmoins par des dimensions très importantes, en particulier pour les hauteurs M4, M7 et la longueur M1. La longueur du corps du calcanéus (M5) est située hors de la variation moderne. En effet, pour Regourdou 2, le corps du calcanéus est particulièrement allongé comme en témoigne le rapport M5/M1. Les calcanéus néandertaliens possèdent un corps volumineux et allongé par rapport aux Hommes modernes (Schmitt 1998).

Pour Raichlen, Armstrong et Liebermann (2011), le corps volumineux des calcanéus néandertaliens entrainerait une consommation énergétique plus importante lors de la course à pied mais serait plus avantageux sur des terrains accidentés ou montagneux. II convient néanmoins de nuancer ces résultats. En effet, la moyenne pour la longueur du corps du calcanéus de notre échantillon de référence néandertalien est plus faible $(59,5 \mathrm{~mm})$ que celle de l'étude précédente $(61,98 \mathrm{~mm})$ se rapprochant ainsi de la moyenne moderne.

Par ailleurs, le calcanéus Regourdou 2 présente toutes les autres caractéristiques néandertaliennes décrites par A. Schmitt (1998). A l'exception des facettes talaires antérieure et moyenne, les surfaces articulaires conservées sont de grandes dimensions (M10 et M12) et les zones d'insertion musculaire sont développées (T1). Ces deux derniers caractères sont également présents sur les 
calcanéus des Hommes anatomiquement modernes du Paléolithique moyen du Proche-Orient et du Paléolithique supérieur.

La plupart des études sur le tarse des Néandertaliens insiste sur une importante robustesse de ces ossements (Trinkaus 1975 ; Rhoads et Trinkaus 1977 ; Gambier 1981, 1982 ; Trinkaus, 1983a ; Schmitt 1998). Celle du calcanéus néandertalien est attestée par ses dimensions générales, ses insertions musculaires développées et ses surfaces articulaires étendues. Les calcanéus néandertaliens possèdent un corps volumineux et allongé par rapport aux Hommes modernes (Schmitt 1998). Si, comme précédemment évoqué, Regourdou 2 présente certains de ces caractères, en revanche, les dimensions liées à sa largeur (M2, M3 et M8) se trouvent plus généralement dans la moyenne néandertalienne. Les indices découlant de ces dimensions (M2/M1, M3/M1 et M8/M7) sont faibles par rapport à la variabilité néandertalienne et moderne ou se situent tout juste à proximité de leurs moyennes (M4/M1). Malgré son aspect volumineux et sa grande taille, le calcanéus Regourdou 2 présente donc des indices ayant
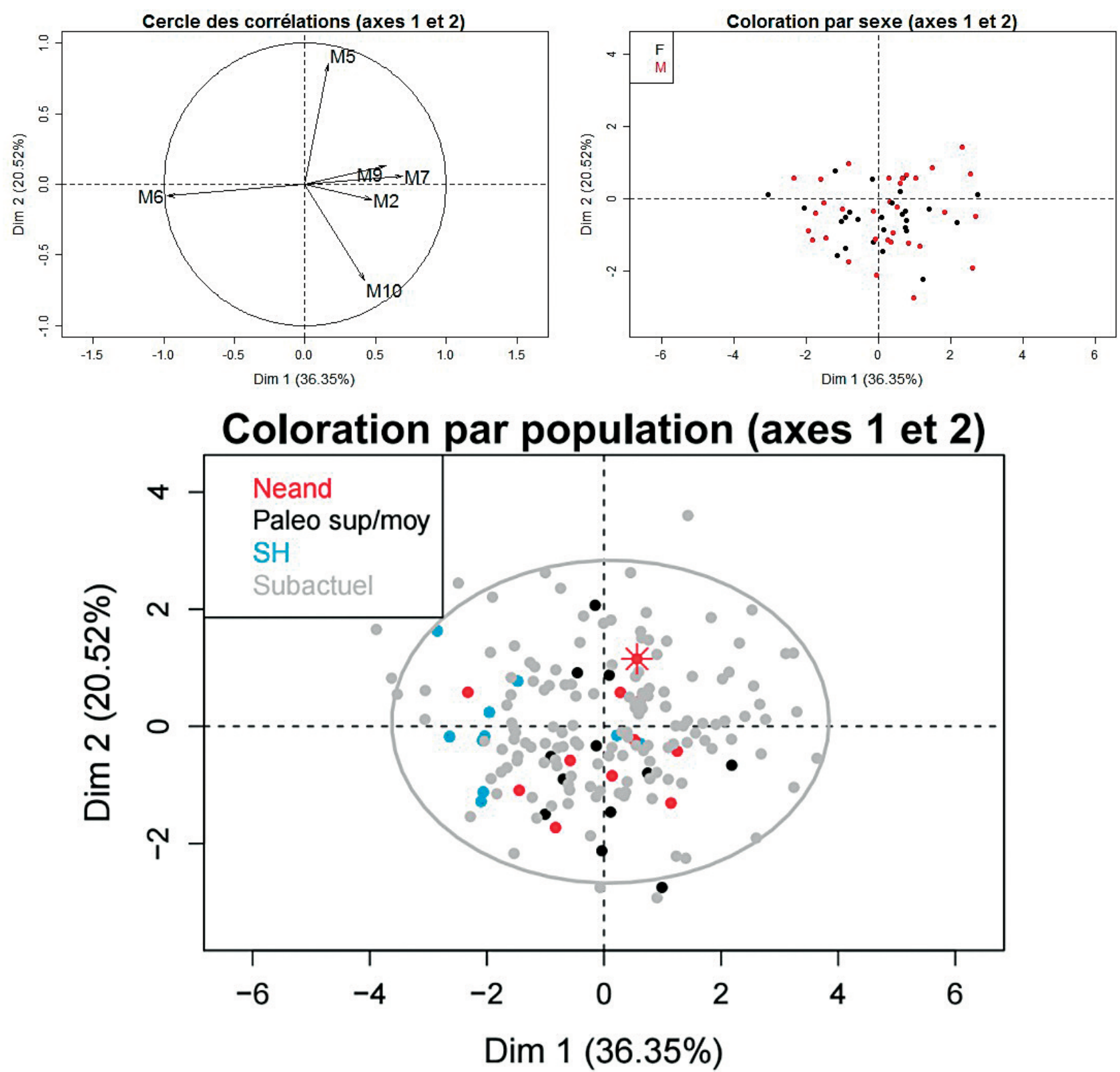

Figure 3 - Analyse en composante principale dont les données ont été double centrées (avec une ellipse de confiance à $95 \%$ pour le groupe subactuel ; l'étoile rouge représente Regourdou 2).

Figure 3 - Double-centered principal component analysis (95\% confidence ellipse for the subactual group; the red star represents Regourdou 2). 


\section{M6}

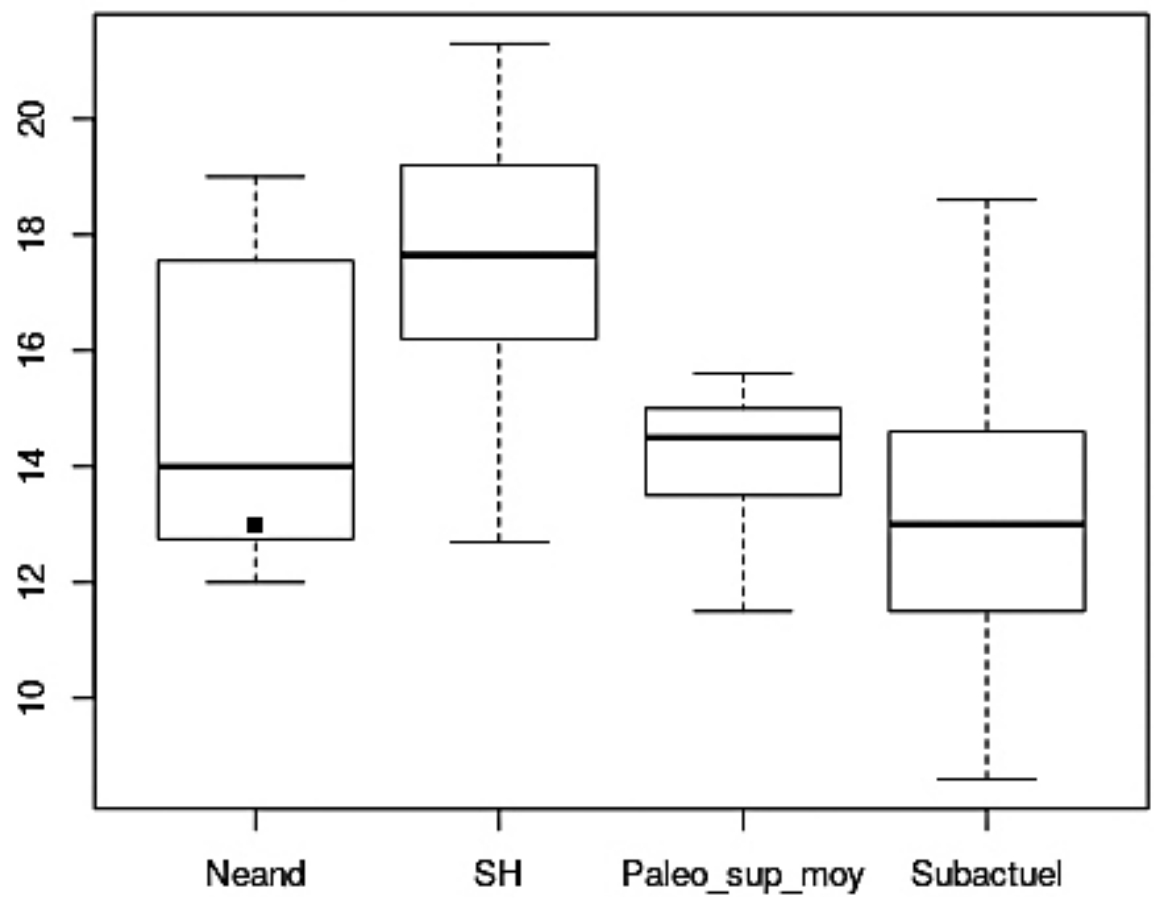

Figure 4 - Boxplot de la variable M6 (Regourdou 2 est représenté par un carré noir, la médiane par le trait plus marqué).

Figure 4 - M6 boxplot (Regourdou 2 is represented by a black square, the median by the darker line).

\begin{tabular}{|l|r|r|r|r|}
\hline & \multicolumn{1}{|c|}{ Neand } & Paleo_sup_moy & \multicolumn{1}{c|}{ SH } & \multicolumn{1}{c|}{ Subactuel } \\
\hline Neand & & 0,744 & 0,791 & 0,035 \\
\hline Paleo_sup_moy & 0,744 & & 0,459 & 0,035 \\
\hline SH & 0,791 & 0,459 & & 0,005 \\
\hline Subactuel & 0,035 & 0,035 & 0,005 & \\
\hline
\end{tabular}

Tableau 6 - p-valeurs issues des comparaisons des groupes deux à deux par tests de Wilcoxon sur la variable M9.

Table 6 - p-values of Wilcoxon test comparing the different groups for M9.

tendance à nuancer sa robustesse générale et qui le distinguent ainsi par ses proportions des autres calcanéus néandertaliens.

D'autres caractères particuliers de Regourdou 2 ont pu être mis en évidence. Ainsi, la morphologie des surfaces articulaires talaires antérieure et moyenne de Regourdou 2 est rare pour le registre néandertalien : 1 observation (Regourdou 2) sur 18 calcanéus (soit 5,6\%). En effet, ses facettes articulaires talaires antérieure et moyenne sont nettement séparées et individualisées, elles sont pour la plupart des autres individus fusionnées (La Chapelle-auxSaints, La Ferrassie 1 et 2, Krapina 240 (Radovčić et al. 1988), Amud, Tabun C1). Pour Kiik-Koba, nous observons une absence de la surface antérieure. En revanche, l'état de conservation de Shanidar 3 (calcanéus droit) et de Spy 2, ne permet pas d'observer leurs surfaces antérieures, leurs surfaces moyennes étant bien délimitées, il n'est pas possible de déterminer si leurs morphologies étaient identiques celle de Regourdou 2 ou bien à celle de Kiik-Koba. Dans notre échantillon moderne, les surfaces sont séparées dans $47 \%$ des cas et fusionnées dans $52 \%$ (dans $1 \%$ des cas, la facette antérieure est absente comme pour Kiik-Koba). Néanmoins, d'autres études ont montré de grandes disparités entre les populations actuelles (Jung et al. 2015). Les conformations se retrouvant dans les mêmes pourcentages chez les adultes et chez les fœtus (Bunning et Barnett 1965), une origine génétique a été envisagée pour ce caractère (Jung et al. 2015).

Les données morphométriques et l'ACP démontrent la grande homogénéité du groupe de La Sima de los Huesos, qui peut s'expliquer par le fait qu'ils proviennent tous du 


\section{M9}

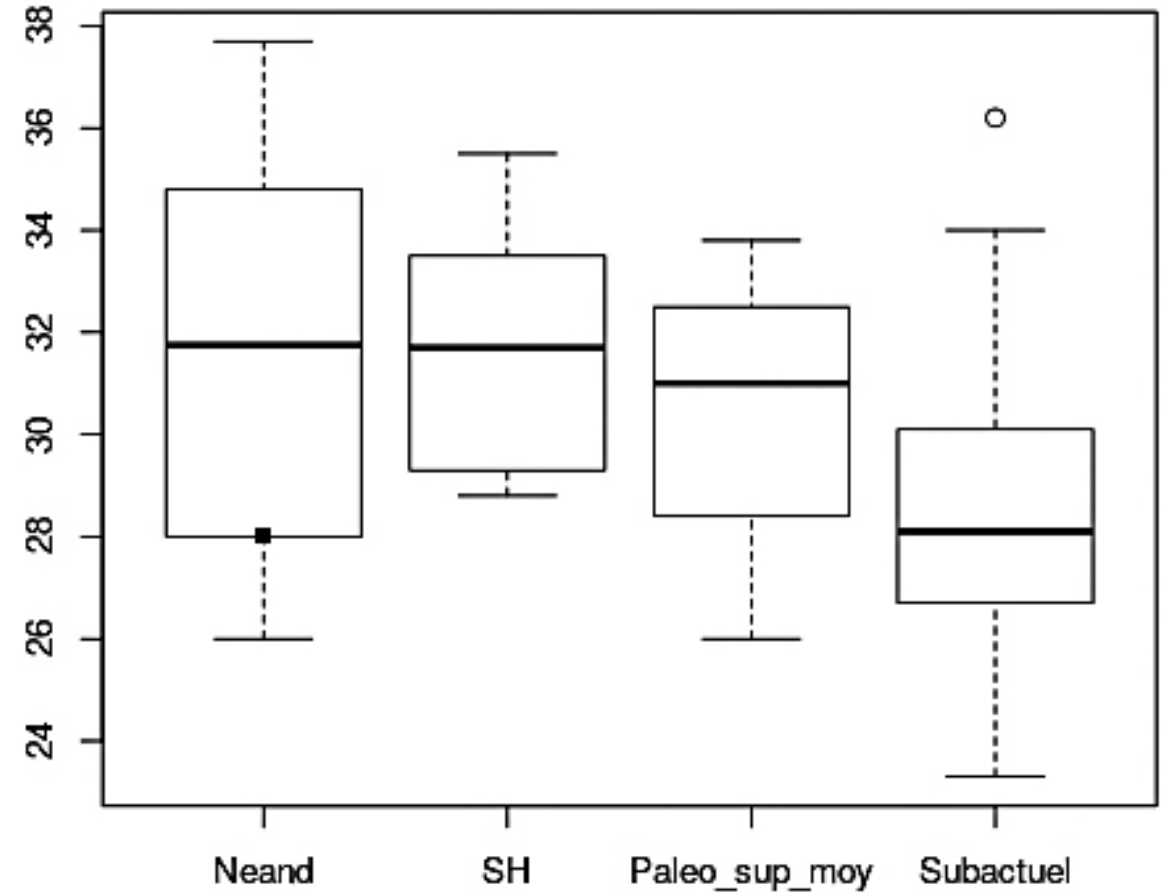

Figure 5 - Boxplot de la variable $\mathrm{M9}$ (Regourdou 2 est représenté par un carré noir, la médiane par le trait plus marqué).

Figure 5 - M9 boxplot (Regourdou 2 is represented by a black square, the median by the darker line). même site et de la même période. On observe également une faible variabilité intra-populationnelle au sein de chacune des populations modernes subactuelles. Par ailleurs, une analyse de variance multivariée non paramétrique (NPMANOVA), menée sur les coordonnées factorielles des individus en retenant les deux premiers axes, montre l'existence de différences significatives entre les groupes étudiés ( $p<0,0001)$. L'étude post-hoc des différences entre toutes les paires de populations montrent que pratiquement toutes nos populations actuelles sont significativement différentes deux à deux (tabl. 7). Les Néandertaliens dits « classiques » présentent quant à eux une plus grande variabilité intra-populationnelle que nos populations modernes prises individuellement.
Une variabilité géographique (nord/sud) au sein de la population néandertalienne a été mise en évidence lors d'études sur les fossiles d'El Sidrón (Rosas et al. 2012) qui concernaient principalement la mandibule. Les analyses génétiques sur cette question ont obtenu des résultats contradictoires (Fabre, Condemi et Degioanni 2009 ; Briggs et al. 2009 ; Prüfer et al. 2014). En ce qui concerne les calcanéus, les ACP ne révèlent pas de différenciations nettes en fonction de l'origine géographique des individus (nord/sud ou Europe/Proche-Orient).

Le calcanéus Regourdou 2 n'étant pas daté et présentant des caractéristiques morphométriques inédites pour le groupe des Néandertaliens, la question d'un rapprochement avec le groupe plus ancien de Sima de los Huesos pouvait alors être posée. Néanmoins les données morphologiques

\begin{tabular}{|l|r|r|r|r|r|r|}
\hline & \multicolumn{1}{|c|}{ Beauvais } & \multicolumn{1}{c|}{ Jau } & \multicolumn{1}{c|}{ Seyssel } & \multicolumn{1}{c|}{ Tel Dan } & \multicolumn{1}{c|}{ Keneh } & \multicolumn{1}{c|}{ Libben } \\
\hline Beauvais & $\mathrm{NA}$ & 0,0431 & $4,00 \mathrm{E}-04$ & $1,00 \mathrm{E}-04$ & $2,00 \mathrm{E}-04$ & $1,00 \mathrm{E}-04$ \\
\hline Jau & 0,0396 & $\mathrm{NA}$ & 0,137 & $6,00 \mathrm{E}-04$ & 0,0125 & 0,0914 \\
\hline Seyssel & $4,00 \mathrm{E}-04$ & 0,1316 & $\mathrm{NA}$ & 0,1772 & $9,00 \mathrm{E}-04$ & 0,337 \\
\hline Tel Dan & $1,00 \mathrm{E}-04$ & $2,00 \mathrm{E}-04$ & 0,1674 & $\mathrm{NA}$ & $1,00 \mathrm{E}-04$ & $3,00 \mathrm{E}-04$ \\
\hline Keneh & $1,00 \mathrm{E}-04$ & 0,0095 & $6,00 \mathrm{E}-04$ & $1,00 \mathrm{E}-04$ & NA & $1,00 \mathrm{E}-04$ \\
\hline Libben & $1,00 \mathrm{E}-04$ & 0,093 & 0,35 & $2,00 \mathrm{E}-04$ & $1,00 \mathrm{E}-04$ & $1,00 \mathrm{E}-04$ \\
\hline
\end{tabular}

Tableau 7 - p-valeurs issues des comparaisons des groupes deux à deux par analyses de variance multivariées non paramétriques (NPMANOVA), effectuées sur les coordonnées des deux premiers axes de l'analyse en composantes principales.

Table 7 - $p$-values of nonparametric multivariate analyses of variance (NPMANOVA) comparing the different populations. The analyses were performed using the two first axes of the PCA as inputs. 
métriques (surfaces articulaires, robustesse, proportions) et multivariées tendent plutôt à éloigner Regourdou 2 des individus d'Atapuerca.

Concernant la taphonomie, cet os présente un aspect concrétionné qui suggère que cette pièce a séjourné à l'air libre. La localisation différentielle de ces concrétions en chou-fleur et les lacunes osseuses présentes sur la face latérale indique que cet os reposait sur sa face latérale et un peu antérieure. Ce vestige montre des atteintes de surface qui se sont produites à l'état sec et dont certaines, notamment la plus importante dont l'aspect évoque l'utilisation d'un outil tranchant de type piochon ou bien truelle, peuvent être mises en relation avec le contexte de la découverte. L'éventualité de dommages causés par des animaux a été envisagée, mais aucune ne possède les caractéristiques d'une morsure ou d'un charognage (Cochard, comm. pers.).

Comme le suggère son état de surface, soit ce calcanéus est à rattacher à un sujet qui se serait décomposé en surface, ou bien alors à un défunt qui aurait évolué en espace colmaté, dont une partie du squelette aurait été par la suite déterrée. La deuxième hypothèse nous semble la plus probable compte tenu de ce que nous connaissons des pratiques funéraires de cette période (Rendu et al. 2014). Nous pouvons toutefois noter que son aspect est bien différent de celui des os de pied de Regourdou 1, dont aucun ne montre des indices de séjour à l'air libre.

\section{8 - Conclusion}

La morphologie du calcanéus Regourdou 2 s'accorde avec son appartenance à un Néandertalien. En effet, l'élongation du corps du calcanéus, les zones d'insertions musculaires développées ainsi que les surfaces articulaires (postérieure et cuboïdienne) étendues sont des caractères connus chez les Néandertaliens. Cependant, cet os possède des caractéristiques qui lui sont propres : de très grandes longueurs, des proportions différentes ainsi que des surfaces antérieure et moyenne de petites tailles et non fusionnées. La robustesse de cet ossement par rapport aux calcanéus modernes est réelle et visible au travers du développement des zones d'insertions musculaire et ligamentaire et de l'étendue des surfaces articulaires. En revanche, elle est moins marquée par rapport aux autres calcanéus néandertaliens en raison de l'étroitesse et des longueurs très importantes de Regourdou 2.

Cette étude a non seulement permis de clarifier l'attribution des différents calcanéus présents à Regourdou et d'attester de la présence de deux individus sur le site mais également d'apporter de nouvelles informations quant à son histoire et sa découverte grâce à l'étude taphonomique. De plus, notre étude permet d'alimenter la base de données des calcanéus néandertaliens et démontre que la variabilité morphologique connue jusqu'alors pour cet os et ce groupe est plus large qu'on ne le pensait.

\section{Remerciements}

Nos remerciements s'adressent à Bernard Vandermeersch qui nous a confié l'étude de ce calcanéus inédit. Nous tenons à remercier Véronique Merlin-Anglade, Conservatrice au Musée d'Art et d'Archéologie du Périgord, et Jean-Jacques Cleyet-Merle, Conservateur au Musée National de Préhistoire des Eyzies-de-Tayac, pour leurs autorisations d'étude du matériel fossile de Regourdou. Nous remercions également David Cochard et Catherine Ferrier (Pacea) pour leurs conseils et orientations sur les atteintes taphonomiques et les concrétions ; Erik Trinkaus (Washington University) pour nous avoir transmis des données métriques inédites et Bruno Maureille (Pacea) pour les discussions que nous avons pu avoir sur le site de Regourdou. Enfin, nous sommes reconnaissants envers Gauthier Devilder (Pacea) pour la conception des planches photographiques.

\section{Références bibliographiques}

ARNOLD L.J., DEMURO M., PARES J.M., ARSUAGA J.L., ARANBURU A., BERMUDEZ de CASTRO J.M., CARBONELL E. 2014 - Luminescence dating and palaeomagnetic age constraint on hominins from Sima de los Huesos, Atapuerca, Spain, Journal of Human Evolution, 67, p. 87-107.

ARSUAGA J.L., CARRETERO J.M., MARTÍNEZ I., GRACIA A. 1991 - Cranial remains and long bones from Atapuerca/lbeas (Spain). Journal of Human Evolution, 20, p. 191-230.

BABA H., ENDO B. 1982 - Postcranial skeleton of the Minatogawa man, in Minatogawa man., Tokyo, Éd. University of Tokyo Press, p. 61-195.

BIDMOS M. A., ASALA S. A. 2003 - Discriminant function sexing of the calcaneus of the South African whites. Journal of Forensic Science 48, p. 1213-1218.

BIDMOS M. A., ASALA S. A. 2004 - Sexual dimorphism of the calcaneus of South African Blacks. Journal of Forensic Science 49, p. 446-450.

BILLY G. 1969 - Le squelette post-crânien de l'homme de Chancelade, L'Anthropologie, 73, p. 207-246.

BILLY G. 1975 - Étude Anthropologique des Restes Humains de l'Abri Pataud., in H.L. Movius dir., Excavation of the Abri Pataud, Les Eyzies (Dordogne), Éd. Peabody Museum of Archaeology and Ethnology, Harvard University, p. 201-305.

BIZOT B., SERRALONGUE J. 1988 - Un édifice funéraire du haut Moyen Age à Seyssel Albigny (Haute-Savoie), Archéologie du Midi médiéval, Vol. 6 (1) p. 25-49.

BONNET R. 1919 - Die Skelete. In : VERWORN M., BONNET R., STEINMANN G. ed. Der diluviale Menschen fund von Obercassel bei Bonn, III, Bergman, Wiesbaden, p. 11-185.

BONIN G.V. 1935 - The Magdalenian Skeleton from CapBlanc in the Field Museum of Natural History: With Nine Plates, Éd. University of Illinois, 76 p. 
BOULE M. 1911-1913 - L'Homme fossile de La Chapelleaux-Saints, Paris : Masson.

BRÄUER G. 1988 - Osteométrie, In : R. Knussman dir., Anthropologie: Handbuch der vergleichenden Biologie des Menschen, Band1, Stuttgart, Éd. Gustav Fischer Verlag, p. $160-232$.

BRIGGS A.W., GOOD J.M., GREEN R.E., KRAUSE J., MARICIC T., STENZEL U., LALUEZA-FOX C., RUDAN P., BRAJKOVIC D., KUCAN Ž., GUSIC I., SCHMITZ R., DORONICHEV V.B., GOLOVANOVA L.V., DE LA RASILLA M., FORTE, J., ROSAS A., PÄÄBO S. 2009 Targeted Retrieval and Analysis of Five Neandertal mtDNA Genomes. Science 325, p. 318-321.

BUNNING P.S., BARNETT C.H. 1965 - A comparison of adult and foetal talocalcaneal articulations. Journal of Anatomy 99, p. 71-76.

CARTRON I., CASTEX D. 2006 - L'occupation d'n ancien îlot de l'estuaire de la Gironde: du temple antique à la chapelle Saint-Siméon (Jau-Dignac et Loirac), Aquitania, t. XXII, p. 253-282.

CHEVALIER N. 1993 - Le cimetière du couvent des Sœurs Grises de Beauvais de la fin du XV ${ }^{*}$ au XVIII siècle, Étude des pratiques funéraires, Mémoire de DEA, Université Bordeaux 1 (Inédit).

CIBERT C., FERMON Y., VALLOD D., MEUNIER F.J. 1999 - Morphological screening of carp Cyprinus carpio: relationship between morphology and fillet yield, Aquatic Living Resources, 12, 1, p. 1-10.

COHEN K.M., GIBBARD P. 2011 -- Regional chronostratigraphical correlation chart for the last 270,000 years: Europe north of the Mediterranean, INQUA, Bern Switzerland.

DE MENDIBURU F. 2017 - agricolae: Statistical Procedures for Agricultural Research. R package version 1.2-6. https://CRAN.R-project.org/package=agricolae.

ENDO B., KIMURA T. 1970 - Postcranial Skeleton of the Amud Man., in SUZUKI H. et TAKAI F. dir., The Amud man and his cave, Éd. Tokyo: Academic Press, p. 231-406.

FABRE V., CONDEMI S., DEGIOANNI A. 2009 - Genetic Evidence of Geographical Groups among Neanderthals, PLOS ONE, 4, 4, e5151.

FÉMOLANT J.-M. 1992 - Beauvais Caserne Taupin « Antenne Universitaire ", Bilan scientifique, Service Régional de l'Archéologie de Picardie, p. 48-50.

FEREMBACH D. 1978 - L'origine des Mésolithiques en France, Bulletins et Mémoires de la Société d'anthropologie de Paris, XIII Série. Tome 5, fascicule 3, p. 231-237.

FRAIPONT J., LOHEST M. 1887 - La race humaine $d u$ Néanderthal ou du Canstadt en Belgique. Recherches ethnographiques sur les ossements humains découverts dans les dépôts quaternaires d'une grotte à Spy et détermination de leur âge géologique. Gand, Archives de Biologie, 7, p. 587-757

GAMBIER D. 1981 - Étude de l'astragale chez les Néandertaliens. Paris, Université Paris VI, 1981, 148 p. thèse.

GAMBIER D. 1982 - Étude ostéométrique des astragales néandertaliens du Regourdou (Montignac, Dordogne). Compte rendu de l'Académie des Sciences de Paris, p. $517-520$

GÓMEZ-OLIVENCIA A., FRANCISCUS R.G., COUTUREVESCHAMBRE C., MAUREILLE B., ARSUAGA J. L. 2012 - The mesosternum of the Regourdou 1 Neandertal revisited. Journal of Human Evolution, 62, 4: 511-519.

GÓMEZ-OLIVENCIA A., COUTURE-VESCHAMBRE C., MADELAINE S., MAUREILLE B. 2013 - The vertebral column of the Regourdou 1 Neandertal. Journal of Human Evolution, 64, p. 582-607.

GRÜN R., STRINGER C. 2000 - Tabun revisited: revised ESR chronology and new ESR and U-series analyses of dental material from Tabun C1, Journal of Human Evolution, 39, 6, p. 601-612.

GUÉRIN G., FROUIN M., TALAMO S., ALDEIAS V., BRUXELLES L., CHIOTTI L., DIBBLE H.L., GOLDBERG P., HUBLIN J.-J., JAIN M., LAHAYE C., MADELAINE S., MAUREILLE B., MCPHERRON S.J.P., MERCIER N., MURRAY A.S., SANDGATHE D., STEELE T.E., THOMSEN K.J., TURQ A.., 2015 - A multi-method luminescence dating of the Palaeolithic sequence of $\mathrm{La}$ Ferrassie based on new excavations adjacent to the La Ferrassie 1 and 2 skeletons. J. Archaeol. Sci. 58, p. 147-166.

HAESAERTS P., BORZIAC I., CHIRICA V., DAMBLON F., KOULAKOVSKA L. 2007 - Cadre stratigraphique et chronologique du Gravettien en Europe centrale, Paleo, 19, p. 31-51.

HARE P. E. 1980 - Organic geochemistry of bone and its relation to the survival of bone in the natural environment, dans Fossils in the Making, Behrensmayer A. K. and HILL A. P., University of Chicago Press, Chicago, p. 208-219.

HEIM J.-L. 1982 - Les hommes fossiles de la Ferrassie Tome 2, Archives de l'Institut de Paléontologie humaine, Paris, Éd. Masson vol. 2.

HENRY-GAMBIER D. 2002 - Les fossiles de Cro-Magnon (Les Eyzies-de-Tayac, Dordogne) : nouvelles données sur leur position chronologique et leur attribution culturelle ", Paleo, 14, p. 201-204.

HERSHKOVITZ, I., SPEIRS M. S., FRAYER D., NADEL D., WISH-BARATZ S., et ARENSBURG B. 1993 - Ohalo II H2: A 19,000-year-old skeleton from a water-logged site at the Sea of Galilee, Israel, American Journal of Physical Anthropology 96, 3, p. 215-234. 
INTRONA F., DIVELLA G., CAMPOBASSO C., DRAGONE M. 1997 - Sex determination by discriminant analysis of Calcanei measurements. Journal of Forensic Science, 42, p. 725-728.

JACOBI, R.M., et HIGHAM T.F.G., 2008 - The "Red Lady" ages gracefully: new ultrafiltration AMS determinations from Paviland, Chronology of the Middle-Upper Paleolithic Transition in Eurasia 55, 5, p.898-907.

JÖRIS O., WENINGER B. 2004 - Coping with the cold: On the climatic context of the Moravian Mid Upper Palaeolithic. The Gravettian along the Danube. The Dolní V stonice Studies 11, p. 57-70.

JUNG M.-H., CHOI B.Y., LEE J.Y., HAN C.S., LEE J.S., YANG Y.C., CHO B.P., 2015 - Types of subtalar joint facets. Surgical Radiologic Anatomy 37, p. 629-638.

KLEIN R.G. 1965 - The Middle Palaeolithic of the Crimea, Arctic Anthropology, 3, 1, p. 34-68.

KOBAYASHI H., HIROSE T., SUGINO M., WATANABE N. 1974 - University of Tokyo radiocarbon measurements $\mathrm{V}$, Radiocarbon, 16, 3, p. 381-387.

LEMÉE É. 2008 (inédit) - Le calcanéus droit de Regourdou 1 dans la variabilité néandertalienne et moderne : étude morphométrique, Mémoire de Master 2, Université Bordeaux 1, Bordeaux, 69 p.

MADELAINE S., MAUREILLE B., CAVANHIÉ N., COUTURE C., BONIFAY E., ARMAND D., BONIFAY M.F., DUDAY H., FOSSE P., VANDERMEERSCH B. 2008 Nouveaux restes humains moustériens rapportés au squelette néandertalien de Regourdou 1 (Regourdou, commune de Montignac, Dordogne, France), Paleo, 20. p. 101-114.

MARIN-MONFORT M.D., SUÑER M., FERNANDEZJALVO Y. 2017 - Characterization of recent marks produced on fossil bone surface during sullegic and trephic processes and their influence on taphonomic studies. Quaternary International sous presse, doi.org/10.1016/ j.quaint.2017.07.039.

MARTIN R., SALLER K. 1956 - Lehrbuch der Anthropologie in systematischer Darstellung, Stuttgart, Éd. G. Fischer. 518 p.

MARTÍNEZ, I., ARSUAGA J.L. 1997 - The temporal bones from Sima de los Huesos Middle Pleistocene site (Sierra de Atapuerca, Spain). A phylogenetic approach. Journal of Human Evolution, 33, p. 283-318.

MATIEGKA J. 1938 - Homo predmostensis. L'homme fossile de Predmosti en Moravie. II. Autres parties du squelette, Prague, Ed. Cesk akademie ved a umení, 91 p.

MAUREILLE B., GÓMEZ-OLIVENCIA A., COUTUREVESCHAMBRE C., MADELAINE S., HOLLIDAY T. 2015a Nouveaux restes humains provenant du gisement de
Regourdou (Montignac-sur-Vézère, Dordogne, France). Paleo, 26, p. 117-138.

MAUREILLE B., HOLLIDAY T., ROYER A., PELLETIER M., MADELAINE S., LACRAMPE-CUYAUBĖRE F., MUTH X., LE GUEUT E., COUTURE-VESCHAMBRE CH., GÓMEZ -OLIVENCIA A., DISCAMPS E., TEXIER J.-P., TURQ A., LAHAYE C. 2015b - Importance des données de terrain pour la compréhension d'un potentiel dépôt funéraire moustérien : le cas du squelette de Regourdou 1 (Montignac-sur-Vézère, Dordogne, France), Paleo, 26, p.139-159.

MCCOWN T.D., KEITH A.B. 1939 - The Stone Age of Mount Carmel: the fossil human remains from the Levalloiso-Mousterian. 2, Éd. Clarendon Press, 390 p.

MEYER V., BRUZEK J., COUTURE-VESCHAMBRE C., MADELAINE S. et MAUREILLE B. 2011 - Un nouveau bassin néandertalien : description morphologique des restes pelviens de Regourdou 1 (Montignac, Dordogne, France). Paleo, 22, p. 207-222.

PABLOS A., MARTINEZ I., LORENZO C., SALA N., GRACIA-TELLEZ A., ARSUAGA J.L. 2014 - Human calcanei from the Middle Pleistocene site of Sima de los Huesos (Sierra de Atapuerca, Burgos, Spain), Journal of Human Evolution. 76, p. $63-76$.

PABLOS A., PANTOJA-PÉREZ A., MARTÍNEZ I., LORENZO C., ARSUAGA J.L. 2015 - Metric and morphological analysis of the foot in the Middle Pleistocene sample of Sima de los Huesos (Sierra de Atapuerca, Burgos, Spain). Quaternary International.

PIVETEAU J. 1963 - La grotte de Regourdou (Dordogne). Annales de Paléontologie, t. XLIX, p. 285-304.

PIVETEAU J. 1964 - La grotte de Regourdou (Dordogne). Paléontologie humaine. Annales de Paléontologie (Vertébrés), t. L, p. 155-194.

PIVETEAU J. 1966 - La grotte de Regourdou (Dordogne). Paléontologie humaine. Annales de Paléontologie (Vertébrés), t. LII, p. 163-194.

PRUFER K., RACIMO F., PATTERSON N., JAY F., SANKARARAMAN S., SAWYER S., HEINZE A., RENAUD G., SUDMANT P.H., DE FILIPPO C. et al. 2014, The complete genome sequence of a Neanderthal from the Altai Mountains, Nature, 505 p. 43-49.

RADOVČIĆ J., SMITH F.H., TRINKAUS E., WOLPOFF M.H. 1988 - The Krapina hominids: an illustrated catalog of skeletal collection, Zagreb, Yugoslavia, Éd. Mladost: Croatian Natural History Museum), 119 p.

RAICHLEN D.A., ARMSTRONG H., LIEBERMAN D.E. 2011 - Calcaneus length determines running economy: Implications for endurance running performance in modern humans and Neandertals, Journal of Human Evolution, 60, 3, p. 299-308. 
RENDU W., BEAUVAL C., CREVECOEUR I., BAYLE P., BALZEAU A., BISMUTH T., BOURGUIGNON L., DELFOUR G., FAIVRE J-Ph., LACRAMPE-CUYAUBERE F., TAVORMINA C., TODISCO D., TURQ A., MAUREILLE B. 2014 - Evidence supporting an intentional Neandertal burial at La Chapelle-aux-Saints, PNAS, 111 (1), p.81-86.

RHOADS, J.G., TRINKAUS, E. 1977 - Morphometrics of the Neandertal talus. American Journal of Physical Anthropology, 46. 1, p. 29-43.

ROSAS A., ESTALRRICH A., GARCIA-TABERNERO A., BASTIR M., GARCIA-VARGAS S., SANCHEZMESEGUER A., HUGUET R., LALUEZA-FOX C., PEÑAMELIAN Á., KRANIOTI E.F., SANTAMARIA D., DE LA RASILLA M., FORTEA J. 2012 - Les Néandertaliens d'El Sidrón (Asturies, Espagne). Actualisation d'un nouvel échantillon. L'Anthropologie, 116, p. 57-76.

SCHMITT A. 1998 - Approche de la variablité du calcaneus néandertalien. Comparaison avec l'Homme moderne, Bulletins et Mémoires de la Société d'anthropologie de Paris, Nouvelle Série., t. 10, fascicule 3-4, p. 273-292.

SCHWARCZ H.P., GRÜN R., VANDERMEERSCH B., BAR-YOSEF O., VALLADAS H., TCHERNOV E. 1988 ESR dates for the hominid burial site of Qafzeh in Israel, Journal of Human Evolution, 17, 8, p. 733-737.

SEMAL P., ROUGIER H., CREVECOEUR I., JUNGELS C., FLAS D., HAUZEUR A., MAUREILLE B., GERMONPRÉ M., BOCHERENS H., PIRSON S., CAMMAERT L., DE CLERCK N., HAMBUCKEN A., HIGHAM T., TOUSSAINT M., VAN DER PLICHT J. 2009 New data on the late Neandertals: Direct dating of the Belgian Spy fossils, American Journal of Physical Anthropology, 138, p. 421-428.

SHANG H., TRINKAUS E. 2010 - The Early Modern Human from Tianyuan Cave, China. College Station TX: Texas A\&M University Press. p. 245.

SLÁDEK V., TRINKAUS E., HILLSON S.W., HOLLIDAY T.W. 2000 - The People of the Pavlovian: Skeletal Catalogue and Osteometrics of the Gravettian Fossil Hominids from Dolní V stonice and Pavlov, Éd. Academy of Sciences of the Czech Republic, Institute of Archeology in Brno. 5. 244 p.

STEELE D. G. 1976 - The estimation of sex on the basis of Sex Assessment from Talus and Calcaneus 47 the talus and calcaneus. American Journal of Physical Anthropology, 45, p.581-588.

STREET M., TERBERGER T. et ORSCHIEDT J. 2006 - A critical review of the German Paleolithic hominin record ». Journal of Human Evolution, 51, 6, P. 551-579.

STRINGER C.B., GRUN R., SCHWARCZ H.P., GOLDBERG P. 1989 - ESR dates for the hominid burial site of Es Skhul in Israel, Nature, 338, 6218, p. 756-758.
TRINKAUS, E. 1975 - A functional analysis of the Neandertal foot. Philadelphia: University of Pennsylvania, 1975,465 p. thèse.

TRINKAUS E. 1983a - Functional Aspects of Neandertal Pedal Remains, Foot \& Ankle International, 3, 6, p. 377-390.

TRINKAUS E. 1983b - The Shanidar Neandertals, Éd. Academic Press. 502 p.

TRINKAUS E. 1996 - The M. Obturator Internus sulcus on Middle and Late Pleistocene human ischia. American Journal of Physical Anthropology, 101, p. 503-513.

TRINKAUS E. 2000 - The human remains from Paviland Cave: Late Pleistocene and Holocene human remains from Paviland Cave. In: ALDHOUSE-GREEN S.H.R. (ed.) Paviland Cave and the 'Red Lady': A Definitive Report. Bristol: Western Academic and Specialist Press Ltd. p. 141-199.

TRINKAUS E., MALEY B., BUZHILOVA A.P. 2008 - Brief communication: Paleopathology of the Kiik-Koba 1 Neandertal, American Journal of Physical Anthropology, 137,1 , p. 106-112.

TRINKAUS E., BUZHILOVA A. P., MEDNIKOVA M. B. DOBROVOLSKAYA M. V. 2014 - The People of Sunghir: Burials, Bodies and Behavior in the Earlier Upper Paleolithic. New York: Oxford University Press. p. 339.

VALLADAS H., MERCIER N., FROGET L., HOVERS E., JORON J.L., KIMBEL W.H., RAK Y. 1999 - TL Dates for the Neanderthal Site of the Amud Cave, Israel, Journal of Archaeological Science, 26, 3, p. 259-268.

VALLOIS H. V. 1965 -- Le sternum néandertalien du Regourdou. Anthropologische Anzeitung, 29, p. 273-289.

VANDERMEERSCH B. 1965 - Position stratigraphique et chronologie relative des restes humains du Paléolithique moyen dans le Sud-Ouest de la France Annales de Paléontologie Vertébrés, 51, 1, p. 63-126.

VANDERMEERSCH B. 1981 - Les hommes fossiles de Qafzeh (Israël), Éd. Éditions du Centre national de la recherche scientifique. $319 \mathrm{p}$.

VANDERMEERSCH B., TRINKAUS E. 1995 - The postcranial remains of the Régourdou 1 Neandertal: the shoulder and arm remains, Journal of Human Evolution, 28, 5, p. 439-476.

VANGHI V., FRISIA S., BORSATO A. 2017 - Genesis and microstratigraphy of calcite coralloids analysed by high resolution imaging and petrography. Sedimentary Geology, 359, 16-28.

VOGEL J.C., WATERBOLK H.T. 1963 - Groningen radiocarbon dates IV, Radiocarbon, 5, 1, p. 163-202.

VOLPATO V., COUTURE C., VANDERMEERSCH B., MACCHIARELLI R. 2005 - Cortical structural geometry of the Regourdou 1 Neandertal upper limb: bilateral 
asymmetry and handedness, Bulletins et Mémoires de la Société d'anthropologie de Paris, t. 17, p. 288-289.

VOLPATO V., COUTURE C., VANDERMEERSCH B., MACCHIARELLI R. 2006 - High-resolution endostructural characterisation of the Regourdou 1 Neandertal proximal arm: bilateral asymmetry and handedness. Terra Nostra 2, p. 140-141. Résumé de communication.
VOLPATO V., COUTURE C., MACCHIARELLI R., VANDERMEERSCH $\quad$ B. $2011 \quad$ - Endostructural characterisation of the Regourdou 1 Neanderthal proximal arm: bilateral asymmetry and handedness. In: CONDEMI S, WENIGER G-C, editors. Continuity and discontinuity in the peopling of Europe: One hundred fifty years of Neanderthal study, Vertebrate Paleobiology and Paleoanthropology. New York, Springer, p. 175-178. 


\section{Annexe 1 - Liste des mesures définies par Martin et Saller (1956)et par Trinkaus (1975).}

\section{Annex 1 - List of measures defined by Martin and Saller (1956) and Trinkaus (1975).}

M1 - Longueur maximale : Distance en projection du point de la tubérosité calcanéenne le plus postérieur au point le plus antérieur du calcanéus sur le bord supérieur de la surface articulaire cuboïdienne.

M1a - Longueur totale : Distance en droite ligne du point de la tubérosité calcanéenne le plus postérieur, au milieu de la surface articulaire cuboïdienne.

M2 - Largeur médiane : Distance en projection du point de la surface articulaire postérieure le plus saillant latéralement au point le plus médial du sustentaculum tali.

M3 - Largeur minimale du corps : distance en projection du point du point le plus rentrant de la face médiale du corps au point correspondant le plus profond de la face latérale.

M4 - Hauteur du calcanéus : distance en projection du point le plus ensellé de la face supérieure concave du corps du calcanéus au point correspondant de la face inférieure, mesuré perpendiculairement au plan sagittal.

M5 - Longueur du corps : distance en droite ligne du point de la tubérosité le plus postérieur, au point le plus bas du bord antérieur de la surface articulaire postérieure.

M6 - Largeur du sustentaculum tali : distance en projection du bord interne de la gouttière du muscle long fléchisseur de l'hallux au point le plus saillant transversalement du sustentaculum tali.

M7 - Hauteur du tuber calcanei : distance en droite ligne du point le plus haut du bord supérieur de la tubérosité au point le plus antérieur et le plus bas du processus médial de la tubérosité calcanéenne.

M8 - Largeur de tubérosité calcanéenne : Distance du bord latéral de la tubérosité au bord médial, perpendiculaire à la hauteur et au milieu de celle-ci.

M9 - Longueur de la surface articulaire dorsale : distance en droite ligne entre les deux extrémités du diamètre longitudinal de la surface articulaire postérieure.

M10 - Largeur de la surface articulaire dorsale : distance entre les deux points les plus saillants des bords latéraux de la surface articulaire postérieure, mesure perpendiculaire au diamètre longitudinal.

M11 - Hauteur de la surface articulaire dorsale : distance en droite ligne du sommet de la courbe longitudinale de la surface articulaire postérieure à la droite du relie les extrémités de cette courbe, c'est-à-dire le diamètre longitudinal.

M12 - Largeur maximale de la surface articulaire cuboïdienne : distance en droite ligne du point le plus haut du bord médial et supérieur au point le plus bas du bord latéral et inférieur de la surface articulaire cuboïdienne.

M13 - Hauteur de la surface articulaire cuboïdienne : distance en doite ligne du point le plus rentrant de la concavité du bord médial au point qui est le plus éloigné sur le bord latéral convexe de la surface articulaire cuboïdienne.

M14 - Angle d'inclinaison de la facette articulaire postérieure : Angle aigu formé par l'axe du calcanéus et le plus grand diamètre de la facette articulaire postérieure.

T1 - Longueur du processus médial : Distance en projection du point le plus postérieur de la tubérosité au point au point antérieur du processus médial. 
